Kyushu J. Math.

Vol. 61, 2007, pp. 155-167

\title{
OKA'S EXTRA ZERO PROBLEM AND APPLICATION
}

\author{
Sachiko HAMANO \\ (Received 29 November 2005, revised 17 January 2006) \\ Dedicated to the memory of Professor Toshio Nishino
}

\begin{abstract}
We solve the extra zero problem in cylindrical domains in $\mathbb{C}^{n}$. We apply it to study domains of holomorphy in $\mathbb{C}^{n}$ which are not rationally convex. This result generalizes Oka's results.
\end{abstract}

\section{Introduction}

Let $D$ be a domain in $\mathbb{C}^{n}$. The collection $(\mathfrak{z})=\left\{\left(f_{p}, U_{p}\right)\right\}_{p \in D}$, where $f_{p}$ is a holomorphic function in a neighborhood $U_{p}$ of $p$ in $D$, is called a (holomorphic) Cousin II distribution in $D$ if, for every $p, q \in D$ with $U_{p} \cap U_{q} \neq \emptyset, f_{p} / f_{q}$ is a non-vanishing holomorphic function in $U_{p} \cap U_{q}$. For a given $(\mathfrak{z})=\left\{\left(f_{p}, U_{p}\right)\right\}_{p \in D}$ in $D$, the Cousin II problem is the problem of finding a holomorphic function $f$ in $D$ such that $f=f_{p} \lambda_{p}$ in $U_{p}$, where $\lambda_{p}$ is a non-vanishing holomorphic function in $U_{p}$. It is not always solvable (see, for example, $[4,8]$ ). Oka posed the following problem concerning it, in 1943 ([6, No. 2, p. 31, Problem 2]).

EXTRA ZERO PROBLEM. Given a Cousin II distribution $(\mathfrak{z})=\left\{\left(f_{p}, U_{p}\right)\right\}_{p \in D}$ in a domain $D$, find a holomorphic function $f$ in $D$ such that $f=f_{p} \alpha_{p}$ in $U_{p}$, where $\alpha_{p}$ is a holomorphic function in $U_{p}$ and $\left\{f_{p}=0\right\} \cap\left\{\alpha_{p}=0\right\}=\emptyset$.

We call $f$ a solution of the extra zero problem for the given data $(\mathfrak{z})$ in $D$. A cylindrical domain $D$ in $\mathbb{C}^{n}$ is the Cartesian product $D_{1} \times \cdots \times D_{n}=\prod_{i=1}^{n} D_{i}$ of some domains $D_{i}$ in $\mathbb{C}_{z_{i}}(i=1, \ldots, n)$.

In this paper, we solve the extra zero problem in cylindrical domains (Theorem 1). As an application, we obtain Theorem 2 which is a generalization of Oka [5] and the example of Stein [7, p. 757].

2000 Mathematics Subject Classification: Primary 32A60, 32B15, 32C25.

Keywords and Phrases: Cousin problems; extra zero problem; intersection number; zero sets of holomorphic functions; cylindrical domain. 
THEOREM 1. We have the following.

(i) Let $D$ be a cylindrical domain $D$ in $\mathbb{C}^{2}$ and let $(\mathfrak{z})$ be a Cousin II distribution in $D$. Then for every cylindrical domain $D_{0} \Subset D$, the extra zero problem for $(\mathfrak{z}) \mid D_{0}$ is solvable.

(ii) If $n \geq 3$, there exist a cylindrical domain $D$ in $\mathbb{C}^{n}$ and a Cousin II distribution (z) in $D$ such that for some cylindrical domain $D_{0} \Subset D$, the extra zero problem for $(\mathfrak{z}) \mid D_{0}$ is not solvable.

Theorem 2. Let (z) be a Cousin II distribution in a cylindrical domain D in $\mathbb{C}^{n}$. Assume that the analytic hypersurface $[(\mathfrak{z})]$ defined by $(\mathfrak{z})$ is irreducible in $D$. If the Cousin II problem for ( $\mathfrak{z})$ is not solvable in $D$, and if, for every cylindrical domain $D_{0} \Subset D$, the extra zero problem for $(\mathfrak{z}) \mid D_{0}$ is solvable, then $G:=D \backslash[(\mathfrak{z})]$ is a domain of holomorphy in $\mathbb{C}^{n}$ which is not rationally convex in $\mathbb{C}^{n}$.

Remark. In the case of $\mathbb{C}^{2}$, the assumption that, for every cylindrical domain $D_{0} \Subset D$, the extra zero problem for $(\mathfrak{z}) \mid D_{0}$ is solvable, does not require Theorem 1.

\section{Preliminaries}

In order to prove Theorems 1 and 2, we use Satz 5 of Stein [8] concerning intersection number. (See Proposition 2.4 below.) To apply Stein's Satz 5 to the case of cylindrical domains in $\mathbb{C}^{n}$, we need the following definition of an idea from Oka's posthumous publication [6, No. 5, p. 111].

Notation. Let $D=\prod_{k=1}^{n} D_{k}$ be a cylindrical domain in $\mathbb{C}^{n}$. We define:

- $\quad$ for $i \in \mathbb{N}(1 \leq i \leq n)$, put $D^{(i)}=\prod_{k=1, \ldots, n ; k \neq i} D_{k}$, and for $z=\left(z_{1}, \ldots, z_{n}\right)$ $\in \mathbb{C}^{n}$, put $z^{(i)}=\left(z_{1}, \ldots, \hat{z_{i}}, \ldots, z_{n}\right) \in \mathbb{C}^{n-1}$ by omitting $z_{i}$;

- $\quad$ for $i, j \in \mathbb{N}(1 \leq i, j \leq n)$, put $D^{(i j)}=\prod_{k=1, \ldots, n ; k \neq i, j} D_{k}$, and for $z \in \mathbb{C}^{n}$, put $z^{(i j)} \in \mathbb{C}^{n-2}$ by omitting $z_{i}$ and $z_{j}$;

- $\quad$ for $p \in D^{(i j)}$ and an analytic hypersurface $S$ in $D$, put $H(p):=\left\{z \in \mathbb{C}^{n}\right.$ : $\left.z^{(i j)}=p\right\}$ and put $S(p):=S \cap H(p)$, which is an analytic surface in $D_{i} \times D_{j}$.

A Cousin II distribution $(\mathfrak{z})$ in $D$ consists of at most countably many irreducible components $\left(S_{v}, e_{v}\right)(v \in \mathbb{N})$, where $e_{v}$ is the vanishing order of the analytic hypersurface $S_{v}$ in $D$. We put the zero set [(z) $]:=\bigcup_{v \in \mathbb{N}} S_{v} \subset D$.

Definition 2.1. (General position) A point $p \in D^{(i j)}$ is in general position with respect to (z) if $H(p) \cap D \not \subset[(\mathfrak{z})]$, and then define the Cousin II distribution $(\mathfrak{z})(p):=(\mathfrak{z}) \cap H(p)=\left\{\left(S_{v}(p), e_{v}\right)\right\}_{v}$ in $D_{i} \times D_{j}$. 
We note that the set of all points in general position with respect to $(\mathfrak{z})$ is open and dense in $D^{(i j)}$.

Consider a real two-dimensional surface $\Gamma_{i j}:=l_{i} \times l_{j}$ in $D_{i} \times D_{j}$, where $l_{k}:[0,1] \rightarrow D_{k}(k=i, j)$ is an (oriented) smooth simple curve.

Definition 2.2. (Transversality) $\Gamma_{i j}$ is transversal to $(\mathfrak{z})(p)$ if:

(i) $\# \Gamma_{i j} \cap[(\mathfrak{z})(p)]<\infty$ and $\left(\Gamma_{i j} \cap[(\mathfrak{z})(p)]\right) \cap \partial \Gamma_{i j}=\emptyset$;

(ii) for every $Q=\left(q_{i}, q_{j}\right) \in \Gamma_{i j} \cap[(\mathfrak{z})(p)]$, some open bi-disk $U_{i}(Q) \times U_{j}(Q)$ of $Q$ contains some restriction $\Gamma_{i j}^{Q}$ of $\Gamma_{i j}$ around $Q$ such that $[(\mathfrak{z})(p)] \mid\left(U_{i}(Q) \times\right.$ $\left.U_{j}(Q)\right)$ is a graph of a univalent $\varphi: U_{j}(Q) \rightarrow U_{i}(Q)$;

(iii) the curve $\varphi\left(l_{j} \cap U_{j}(Q)\right)$ traverses $l_{i} \cap U_{i}(Q)$ at $q_{i}$.

Then put $s\left([(\mathfrak{z})(p)], \Gamma_{i j}^{Q}\right):=1$ if $\varphi\left(l_{j} \cap U_{j}(Q)\right)$ traverses $l_{i} \cap U_{i}(Q)$ at $q_{i}$ from the right to the left, and otherwise $s\left([(\mathfrak{z})(p)], \Gamma_{i j}^{Q}\right):=-1$, and also define, when $Q \in S_{v}(p)$,

$$
I\left((\mathfrak{z})(p), \Gamma_{i j}^{Q}\right):=s\left([(\mathfrak{z})(p)], \Gamma_{i j}^{Q}\right) \cdot e_{\nu}
$$

Clearly, $I\left((\mathfrak{z})(p), \Gamma_{i j}^{Q}\right)$ is independent of choices of $U_{i}(Q) \times U_{j}(Q)$, and hence well defined.

Definition 2.3. (Intersection number) The intersection number of $(\mathfrak{z})(p)$ and $\Gamma_{i j}$ is defined by

$$
I\left((\mathfrak{z})(p), \Gamma_{i j}\right):=\sum_{Q \in \Gamma_{i j} \cap[(\mathfrak{z})(p)]} I\left((\mathfrak{z})(p), \Gamma_{i j}^{Q}\right) .
$$

For homotopy classes $\left[\gamma_{k}\right]$ of simple closed curves in $D_{k}(k=i, j)$, we note that there exist smooth representatives $\gamma_{k}(k=i, j)$ such that $\Gamma_{i j}=\gamma_{i} \times \gamma_{j}$ is transversal to $(\mathfrak{z})(p)$ for every $p \in D^{(i j)}$ in general position with respect to $(\mathfrak{z})$, and $I\left((\mathfrak{z})(p), \Gamma_{i j}\right)$ is independent of choices of such both $\gamma_{k}(k=i, j)$ and $p$. Hence, the intersection number of $(\mathfrak{z})$ and $\left(\left[\gamma_{i}\right],\left[\gamma_{j}\right]\right)$ is defined by $I\left((\mathfrak{z}),\left[\gamma_{i}\right],\left[\gamma_{j}\right]\right):=I\left((\mathfrak{z})(p), \Gamma_{i j}\right)$. Then we have the following.

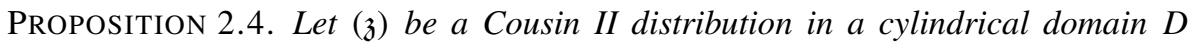
in $\mathbb{C}^{n}$. Then (z) has a solution of the Cousin II problem in D if and only if $I\left((\mathfrak{z}),\left[\gamma_{i}\right],\left[\gamma_{j}\right]\right)$ vanishes for each pair $i, j(1 \leq i, j \leq n ; i \neq j)$, where $\left[\gamma_{k}\right]$ is every homotopy class of a simple closed curve in $D_{k}(k=i, j)$. 


\section{Proof of Theorem 1}

Let $D_{1}, D_{2}$ be domains bounded by a finite number (at least two) of simple closed curves in $\mathbb{C}_{z}, \mathbb{C}_{w}$, respectively. The boundary components of $D_{1}$ is $C_{0}, C_{1}, \ldots, C_{m}$, where $C_{0}$ is the outer boundary; $\partial D_{1}=C_{0}-\sum_{i=1}^{m} C_{i}$. The boundary components of $D_{2}$, as well as $D_{1}$, are $\partial D_{2}=\tilde{C}_{0}-\sum_{j=1}^{n} \tilde{C}_{j}$. For all homotopy classes $\left[\gamma_{i}\right] \times\left[\tilde{\gamma}_{j}\right]$ $(i=1, \ldots, m ; j=1, \ldots, n)$ in $D:=D_{1} \times D_{2}$, where $\gamma_{i} \sim C_{i}\left(\gamma_{i}\right.$ is homotopic to $\left.C_{i}\right)$ in $D_{1}$ and $\tilde{\gamma}_{j} \sim \tilde{C}_{j}$ in $D_{2}$, we consider $\Gamma_{i j}:=\gamma_{i} \times \tilde{\gamma}_{j}$ in $D$.

LEMMA 3.1. Under the above notation, there exist analytic surfaces $\sigma_{i j}^{+}, \sigma_{i j}^{-}$in $D$ such that the intersection number $I\left(\sigma_{i j}^{ \pm},\left[\gamma_{i}\right],\left[\tilde{\gamma}_{j}\right]\right)= \pm 1$, respectively, and $I\left(\sigma_{i j}^{ \pm},\left[\gamma_{h}\right],\left[\tilde{\gamma}_{k}\right]\right)=0$ for $(h, k) \neq(i, j)$.

Proof. Fix $i, j(i=1, \ldots, m ; j=1, \ldots, n)$. By the well-known theorem for conformal mapping theory (see, for example, [9]), there exists a conformal mapping $S_{i}: D_{1} \rightarrow \Delta_{i}^{*}$, where $\Delta_{i}^{*}$ is an annulus $\Delta_{i}:=\left\{r_{i}<|x|<1\right\}$ with $m-1$ radial slits, such that $\left|S_{i}\left(C_{0}\right)\right|=1,\left|S_{i}\left(C_{i}\right)\right|=r_{i}$, and the other contours are flattened into radial slits with two sides, as usual. Similarly, a conformal mapping $\tilde{S}_{j}: D_{2} \rightarrow \tilde{\Delta}_{j}^{*}$ also exists, where $\tilde{\Delta}_{j}^{*}$ is $\tilde{\Delta}_{j}:=\left\{\tilde{r}_{j}<|y|<1\right\}$ with $n-1$ radial slits, such that $\left|\tilde{S}_{j}\left(\tilde{C}_{0}\right)\right|=1$ and $\left|\tilde{S}_{j}\left(\tilde{C}_{j}\right)\right|=\tilde{r}_{j}$. Put $\Delta_{i j}:=\Delta_{i} \times \tilde{\Delta}_{j}$ in $\mathbb{C}_{x} \times \mathbb{C}_{y}$, and $\Delta_{i j}^{*}:=\Delta_{i}^{*} \times \tilde{\Delta}_{j}^{*}$ which is analytically equivalent to $D$.

Let $l_{i}:=\left\{r e^{i \theta} \in \mathbb{C}_{x} \mid r_{i} \leq r \leq 1, \theta=0\right\}$ be a radial segment from $|x|=r_{i}$ to $|x|=1$ of $\Delta_{i}$. We can assume that $m-1$ and $n-1$ radial slits do not lie on $l_{i}$ and $\left\{\tilde{r}_{j} \leq y \leq 1\right\}$, respectively. Fix an argument $\alpha$ such that $0<\alpha<K$, where $K=\min \left\{\arg \left\{\tilde{S}_{j}\left(\tilde{C}_{k}\right)\right\} \mid k \neq 0, j\right\}>0$. Let $\tilde{l}_{j}:=\left\{\rho e^{i \varphi} \in \mathbb{C}_{y} \mid \rho=\tilde{r}_{j}, 0 \leq \varphi \leq \alpha\right\}$ be a circular arc (clockwise) in the positive direction with respect to $\tilde{\Delta}_{j}$ and let $\tilde{\delta}_{j}:=\left\{\rho e^{i \varphi} \in \mathbb{C}_{y} \mid \tilde{r}_{j}<\rho<1,0<\varphi<\alpha\right\}$ be a domain of sector in $\tilde{\Delta}_{j}$. We then have a one-to-one conformal mapping $\Phi: \tilde{\delta}_{j} \rightarrow \mathcal{R}$, where $\mathcal{R}$ is a covering Riemann surface over $\Delta_{i}$ with relative boundaries: $\mathcal{R}=\left\{r e^{i \theta} \in \mathbb{C}_{x} \mid r_{i}<r<1,0<\theta<\beta\right\}$, where $\beta=\left(\log r_{i}\right)\left(\log \tilde{r}_{j}\right) / \alpha$ and $l_{i}=\Phi\left(\tilde{l}_{j}\right)$; in fact, $x=\Phi(y), y \in \tilde{\delta}_{j}$ is obtained by composing functions $v=\log y, u=\sqrt{-1}\left(-v \log r_{i} / \alpha+\beta\right), x=e^{u}$. Set $\Sigma_{i j}^{+}:=\left\{(x, y) \in \Delta_{i j} \mid x-\Phi(y)=0\right\}$. Since $\partial \Sigma_{i j}^{+} \subset \partial \Delta_{i j}, \Sigma_{i j}^{+}$is an analytic surface in $\Delta_{i j}$. Hence, we define an analytic surface pulled back $\Sigma_{i j}^{+} \mid \Delta_{i j}^{*}$ to $D$ as $\sigma_{i j}^{+}$, that is,

$$
\sigma_{i j}^{+}:=\left\{(z, w) \in D \mid S_{i}(z)-\Phi\left(\tilde{S}_{j}(w)\right)=0\right\} .
$$

We first show that $I\left(\sigma_{i j}^{+}, \Gamma_{i j}\right)=+1$ : Pick up an oriented closed $\tilde{\gamma}_{j}$ which is sufficiently close to $\tilde{C}_{j}$, so that $\tilde{S}_{j}\left(\tilde{\gamma}_{j}\right)$ is close to $|y|=\tilde{r}_{j} . \quad \Phi\left(\tilde{S}_{j}\left(\tilde{\gamma}_{j}\right) \cap \tilde{\delta}_{j}\right)$ 
is a transversal curve from $|x|=1$ to $|x|=r_{i}$ in $\Delta_{i}$. Then the oriented curve $S_{i}^{-1}\left(\Phi\left(\tilde{S}_{j}(w)\right)\right), w \in \tilde{\gamma}_{j} \cap \tilde{S}_{j}^{-1}\left(\tilde{\delta}_{j}\right)$, traverses $\gamma_{i}$ from the right to the left. Therefore, $I\left(\sigma_{i j}^{+}, \Gamma_{i j}\right)=+1$. We next show that $I\left(\sigma_{i j}^{+}, \Gamma_{h k}\right)=0$. In the case when $\gamma_{h} \sim C_{h}$ ( $\neq C_{i}$ ), the intersection number of the closed curve $S_{i}\left(\gamma_{h}\right)$ and the transversal curve $\Phi\left(\tilde{S}_{j}\left(\tilde{\gamma}_{j}\right) \cap \tilde{\delta}_{j}\right)$ is canceled in total. Thus, $I\left(\sigma_{i j}^{+}, \Gamma_{h j}\right)=0$. In the case when $\tilde{\gamma}_{k} \sim \tilde{C}_{k}$ $\left(\neq \tilde{C}_{j}\right)$, since we can take $\tilde{\gamma}_{k}$ which is close to $\tilde{C}_{k}$ such that $\tilde{S}_{j}\left(\tilde{\gamma}_{k}\right) \subset \tilde{\Delta}_{j} \backslash \tilde{\delta}_{j}$, $\sigma_{i j}^{+} \cap \Gamma_{i k}=\emptyset$. Hence, $I\left(\sigma_{i j}^{+}, \Gamma_{i k}\right)=0$.

Similarly, let $m_{j}:=\left\{\rho e^{i \varphi} \in \mathbb{C}_{y} \mid \rho=1,0 \leq \varphi \leq \alpha\right\}$ be a circular arc in the positive direction with respect to $\tilde{\Delta}_{j}$, and consider a one-to-one conformal mapping $\hat{\Phi}: \tilde{\delta}_{j} \rightarrow \hat{\mathcal{R}}$ with $l_{i}=\hat{\Phi}\left(m_{j}\right)$. Then $\sigma_{i j}^{-}:=\left\{(z, w) \in D \mid S_{i}(z)-\hat{\Phi}\left(\tilde{S}_{j}(w)\right)=0\right\}$ satisfies the lemma's conditions.

Lemma 3.2. Let $G \subset \mathbb{C}_{z} \times \mathbb{C}_{w}$ be a domain and let $G_{0} \Subset G$. Let $(\mathfrak{z})=$ $\left\{\left(f_{p}, U_{p}\right)\right\}_{p \in G}$ be a Cousin II distribution in $G$. Then there exists a continuous function in some neighborhood of $[(\mathfrak{z})] \mid G_{0}$ with prescribed zero $(\mathfrak{z}) \mid G_{0}$, where $[(\mathfrak{z})]$

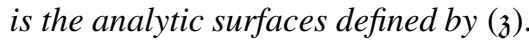

Proof. If necessary, make a coordinates transformation in $\mathbb{C}^{2}$. Then we may assume that $[(\mathfrak{z})]$ satisfies the Weierstrass condition for $w$ at any point on $[(\mathfrak{z})]$ (cf. [2]). Take $G_{1}$ such that $G_{0} \Subset G_{1} \Subset G$. We simply write anew $(\mathfrak{z}):=(\mathfrak{z}) \mid G_{1}$. Then $(\mathfrak{z})$ consists of a finite number of pairs $\left(S_{v}, e_{v}\right)(v=1, \ldots, n)$, where $e_{v}$ is the vanishing order of the irreducible analytic surface $S_{v}$ in $G_{1}$, and $[(\mathfrak{z})]:=\bigcup_{v=1}^{n} S_{v} \subset G_{1}$. Let $p_{i}=\left(z_{i}, w_{i}\right)(i=1, \ldots, m ; m<\infty)$ be the set of singular points at which $[(\mathfrak{z})]$ is locally reducible. Put $[(\mathfrak{z})]^{*}=[(\mathfrak{z})] \backslash\left\{p_{i}\right\}_{i=1}^{m}$ and consider the (analytic) projection $\pi$ from $[(\mathfrak{z})]^{*}$ to $\mathbb{C}_{z}$, so that $\pi\left([(\mathfrak{z})]^{*}\right)$ defines a finitely sheeted Riemann surface $\mathcal{R}$ which might have a finite number of branch points $a_{1}, \ldots, a_{m^{\prime}}$. Note that $[(\mathfrak{z})]^{*}$ and $\mathcal{R}$ are one to one through $\pi$, and $\mathcal{R}$ consists of disjoint connected non-compact Riemann surfaces $\mathcal{R}_{v}(v=1, \ldots, n)$. We have, for each $v=1, \ldots, n$,

$$
[(\mathfrak{z})]^{*}: w=\xi_{v}(z), \quad z \in \mathcal{R}_{v}
$$

where $\xi_{v}$ is a single-valued holomorphic function on $\mathcal{R}_{v}$. Let $p_{j}^{\prime} \in[(\mathfrak{z})]^{*}$ be the point corresponding to the branch point $a_{j} \in \mathcal{R}$, i.e. $\pi\left(p_{j}^{\prime}\right)=a_{j}\left(j=1, \ldots, m^{\prime}\right)$. For the sake of convenience, we write $p_{j}^{\prime}=p_{m+j}\left(j=1, \ldots, m^{\prime}\right)$ and $m+m^{\prime}=m^{\prime \prime}$.

We first give a solution of the given $(\mathfrak{z})$ near $p_{i}\left(i=1, \ldots, m^{\prime \prime}\right)$ in $G_{1}$. At each point $p_{i}=\left(z_{i}, w_{i}\right)$, take a small bi-disk

$$
\Lambda_{i}:=\Delta_{i} \times \Gamma_{i}=\left\{\left|z-z_{i}\right|<2 r_{i}\right\} \times\left\{\left|w-w_{i}\right|<\rho_{i}\right\} \Subset G_{1}
$$


such that those $m^{\prime \prime}$ bi-disks $\left\{\Lambda_{i}\right\}_{i=1, \ldots, m^{\prime \prime}}$ are disjoint and $\left(\Delta_{i} \times \partial \Gamma_{i}\right) \cap[(\mathfrak{z})]=\emptyset$, $\left(\left\{z_{i}\right\} \times \Gamma_{i}\right) \cap[(\mathfrak{z})]=\left\{p_{i}\right\}$. Then Weierstrass' preparation theorem implies

$$
f_{p_{i}}=\omega_{i} \prod_{h=1}^{l_{i}}\left[P_{h}\right]^{e_{v}}
$$

where each $P_{h}$ is an irreducible distinguished pseudopolynomial in $w$ at $\left(z_{i}, w_{i}\right)$ and $\omega_{i}$ is a non-vanishing holomorphic function for $(z, w)$ in $\Lambda_{i}$. Here, $\omega_{i}, l_{i}, P_{h}, e_{v}$ depend on $i$. Moreover, $P_{h}=\prod_{\mu=1}^{k}\left(w-\xi_{v}^{\mu}\right)$, where each $\xi_{v}^{\mu}$ is some branch of the same $w=\xi_{v}$ in (3.1). Put $\mathfrak{P}_{i}=\prod_{h=1}^{l_{i}}\left[P_{h}\right]^{e_{v}}$ and consider the pairs $\left(\mathfrak{P}_{i}, \Lambda_{i}\right)\left(i=1, \ldots, m^{\prime \prime}\right)$. Then they become a solution of $(\mathfrak{z})$ in the neighborhood $\Lambda:=\bigcup_{i=1}^{m^{\prime \prime}} \Lambda_{i}$ of $\bigcup_{i=1}^{m^{\prime \prime}}\left\{p_{i}\right\}$.

Putting for each $i=1, \ldots, m^{\prime \prime}$,

$$
\delta_{i}:=\left\{\left|z-z_{i}\right|<r_{i}\right\} \subset \Delta_{i}, \quad \lambda_{i}:=\delta_{i} \times \Gamma_{i} \subset \Lambda_{i}, \quad \lambda:=\bigcup_{i=1}^{m^{\prime \prime}} \lambda_{i} \subset \Lambda .
$$

We next give a solution of $(\mathfrak{z})$ in a neighborhood of $[(\mathfrak{z})] \cap\left(G_{0} \backslash \lambda\right)$ in $G_{1}$. We simply set $[(\mathfrak{z})]^{e}:=[(\mathfrak{z})] \cap\left(G_{1} \backslash \lambda\right)$, which consists of $n$ disjoint irreducible analytic surfaces $[(\mathfrak{z})]_{v}^{e}(v=1, \ldots, n)$. Since $[(\mathfrak{z})]^{e} \subset[(\mathfrak{z})]^{*}$, we put $\mathcal{R}^{e}=\pi\left([(\mathfrak{z})]^{e}\right)$, which consists of $n$ disjoint connected non-compact Riemann surfaces $\mathcal{R}_{v}^{e}:=\pi\left([(\mathfrak{z})]_{v}^{e}\right) \subset \mathcal{R}_{v}$ $(v=1, \ldots, n)$ without branch points over $\mathbb{C}_{z}$. From (3.1), we also have

$$
[(\mathfrak{z})]_{v}^{e}: \quad w=\xi_{v}(z), \quad z \in \mathcal{R}_{v}^{e} .
$$

Take $G^{\prime}$ such that $G_{0} \Subset G^{\prime} \Subset G_{1}$ and $\left(G_{1} \backslash G^{\prime}\right) \cap \Lambda=\emptyset$. Put $\left(\mathcal{R}_{v}^{e}\right)^{\prime}=\pi\left([(\mathfrak{z})]_{\nu}^{e} \cap G^{\prime}\right)$ $\subset \mathcal{R}_{v}^{e}$. We take a sufficiently thin tubular neighborhood $V_{v}\left(\subset G_{1}\right)$ of each $[(\mathfrak{z})]_{v}^{e} \cap G_{0}$ $(v=1, \ldots, n)$ :

$$
V_{v}=\bigcup_{z \in\left(\mathcal{R}_{v}^{e}\right)^{\prime}}\left(z,\left\{\left|w-\xi_{v}(z)\right|<\varepsilon\right\}\right)
$$

such that those $n$ tubular neighborhoods $\left\{V_{v}\right\}_{\nu=1, \ldots, n}$ are disjoint in $G_{1}$. Therefore, if we put

$$
f_{v}=\left(w-\xi_{v}\right)^{e_{v}} \quad \text { in } V_{v}
$$

and consider the pairs $\left(f_{\nu}, V_{v}\right)(v=1, \ldots, n)$, then they become a solution of $(\mathfrak{z})$ in the neighborhood $\mathcal{V}:=\bigcup_{\nu=1}^{n} V_{v}$ of $[(\mathfrak{z})] \cap\left(G_{0} \backslash \lambda\right)$.

We consider, for each $i=1, \ldots, m^{\prime \prime}, A_{i}:=\Delta_{i} \backslash \delta_{i}=\left\{z \in \Delta_{i}\left|r_{i}<\right| z-z_{i} \mid\right.$ $\left.<2 r_{i}\right\}$ and $\mathcal{A}_{i}:=A_{i} \times \Gamma_{i}$. If necessary, take a smaller $\varepsilon>0$. Then we may assume that $V_{v} \cap\left(A_{i} \times \partial \Gamma_{i}\right)=\emptyset$, so that $V_{v} \cap\left(A_{i} \times \mathbb{C}_{w}\right) \subset \mathcal{A}_{i}$. Hence, for each $V_{v}$ and $\Lambda_{i}$ 
such that $V_{v, i}:=V_{v} \cap \Lambda_{i}=V_{v} \cap \mathcal{A}_{i} \neq \emptyset\left(v=1, \ldots, n ; i=1, \ldots, m^{\prime \prime}\right)$, we see that $\mathfrak{P}_{i} / f_{v}$ is a non-zero holomorphic function on $V_{v, i}$. Let $h_{v, i}$ be the restriction of $\mathfrak{P}_{i} / f_{v}$ onto $[(\mathfrak{z})]_{v, i}^{e}:=[(\mathfrak{z})]_{v}^{e} \cap V_{v, i}$. We define $h_{v}:=h_{v, i}$ in $[(\mathfrak{z})]_{v, i}^{e}\left(i=1, \ldots, m^{\prime \prime}\right)$. Put $\mathcal{E}_{v}:=\pi\left(\bigcup_{i=1}^{m^{\prime \prime}}[(\mathfrak{z})]_{v, i}^{e}\right) \subset \mathcal{R}_{v}^{e}$ and regard $h_{v}$ as a non-zero holomorphic function on $\mathcal{E}_{v}$. We remark that, since $\mathcal{R}_{v}^{e}$ is non-compact Riemann surface and $\mathcal{R}_{v}^{e} \backslash \mathcal{E}_{v}$, which consists of one component, is not relatively compact in $\mathcal{R}_{v}^{e}$, it follows that the pair $\left(\mathcal{R}_{v}^{e}, \mathcal{E}_{v}\right)$ satisfies Runge's theorem. Moreover, there exists a non-zero holomorphic function $\tilde{h}_{v}$ on $\mathcal{R}_{v}^{e}$ (which is regarded as a function on $[(\mathfrak{z})]_{v}^{e}$ ) such that $\tilde{h}_{v} \approx h_{v}$ on $\mathcal{E}_{v}$ (approximates sufficiently close).

For $v=1, \ldots, n, i=1, \ldots, m^{\prime \prime}$, if we put $B_{v, i}:=V_{v} \cup \lambda_{i}, B:=$ $\bigcup_{v=1}^{n} \bigcup_{i=1}^{m^{\prime \prime}} B_{v, i}$, then $B=\left(\bigcup_{\nu=1}^{n} V_{\nu}\right) \cup\left(\bigcup_{i=1}^{m^{\prime \prime}} \lambda_{i}\right)=\mathcal{V} \cup \lambda$ is a neighborhood of $[(\mathfrak{z})] \cap G_{0}$. For each $B_{v, i}$, if we define $F:=F_{v, i}$, where

$$
F_{v, i}:= \begin{cases}\mathfrak{P}_{i} & \text { in } \lambda_{i}, \\ \mathfrak{P}_{i} \cdot \frac{2 r_{i}-\left|z-z_{i}\right|}{r_{i}}+\sqrt{-1} \cdot f_{v} \cdot \tilde{h}_{v} \cdot \frac{\left|z-z_{i}\right|-r_{i}}{r_{i}} & \text { in } V_{v, i}, \\ \sqrt{-1} \cdot f_{v} \cdot \tilde{h}_{v} & \text { in } V_{v} \backslash V_{v, i},\end{cases}
$$

then $F$ is a well-defined single-valued continuous function on $B$ with the prescribed zero $(\mathfrak{z}) \mid G_{0}$; in fact, it is clear that $F$ is a continuous function in $B$ and has the prescribed zero $(\mathfrak{z}) \mid G_{0}$ in $B \backslash \bigcup V_{\nu, i}$. It remains to prove that $F=0$ is $(\mathfrak{z}) \mid G_{0}$ in each $V_{v, i}$. In $V_{v, i}$, we consider

$$
F_{v, i}=f_{v} \cdot\left(\frac{\mathfrak{P}_{i}}{f_{v}} \cdot \frac{2 r_{i}-\left|z-z_{i}\right|}{r_{i}}+\sqrt{-1} \cdot \tilde{h}_{v} \cdot \frac{\left|z-z_{i}\right|-r_{i}}{r_{i}}\right)
$$

and denote the second part by $\varphi$, i.e. $F_{v, i}=f_{v} \cdot \varphi$ in $V_{v, i}$. Since $\mathfrak{P}_{i} / f_{v} \mid[(\mathfrak{z})]_{v, i}^{e}=h_{v, i}$, $h_{v} \mid[(\mathfrak{z})]_{v, i}^{e}=h_{v, i}$, and $\tilde{h}_{v} \approx h_{v}$ on $\mathcal{E}_{v}\left(\supset \pi\left([(\mathfrak{z})]_{v, i}^{e}\right)\right)$, it follows that $\tilde{h}_{v} \approx \mathfrak{P}_{i} / f_{v}$ on $[(\mathfrak{z})]_{v, i}^{e}$. Thus, $\varphi \mid[(\mathfrak{z})]_{v, i}^{e} \neq 0$. If we take a smaller $\varepsilon>0$, then $\varphi \neq 0$ in $V_{v, i}$.

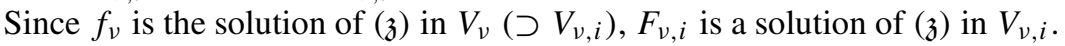

Proof of case (i) in Theorem 1. Given a Cousin II distribution ( $\mathfrak{z}$ ) in a cylindrical domain $D$ in $\mathbb{C}^{2}$ and take a relatively compact cylindrical domain $D_{0}=D_{1}^{0} \times D_{2}^{0}$ in $D$. Without loss of generality, we may assume that each component $D_{k}^{0}(k=1,2)$ of $D_{0}$ is bounded by a finite number of piecewise smooth simple closed curves. Take a cylindrical domain $D^{*}\left(D_{0} \Subset D^{*} \Subset D\right)$ such that each component of $D^{*}$, whose connectivity is the same as that of $D_{k}^{0}(k=1,2)$, is bounded by a finite number of piecewise smooth simple closed curves. Let $\gamma_{i}(i=1, \ldots, m)$ 
and $\tilde{\gamma}_{j}(j=1, \ldots, n)$ be base cycles in $D_{1}^{0}$ and $D_{2}^{0}$, respectively. For each $\Gamma_{i j}:=\gamma_{i} \times \tilde{\gamma}_{j}$ in $D_{0}$, put $e_{i j}:=I\left((\mathfrak{z}), \Gamma_{i j}\right)$ in $D$. Then we can make a Cousin II distribution $\left(\mathfrak{z}^{*}\right)$ in $D^{*}$ such that $I\left(\left(\mathfrak{z}^{*}\right), \Gamma_{i j}\right)=-e_{i j}$ in the following way; if $e_{i j}>0$, then we consider a Cousin II distribution in $D^{*}$ consisting of the analytic surface $\sigma_{i j}^{-}$in $D^{*}$ which satisfies the conditions of Lemma 3.1 and the vanishing order $+e_{i j}$ of $\sigma_{i j}^{-}$. If $e_{i j}<0$, then we consider a Cousin II distribution in $D^{*}$ composed of the analytic surface $\sigma_{i j}^{+}$in $D^{*}$ which satisfies the conditions of Lemma 3.1 and the vanishing order $-e_{i j}$ of $\sigma_{i j}^{+}$. If $e_{i j}=0$, then we consider a constant function 1 in $D^{*}$ as a Cousin II distribution. Then we define the Cousin II distribution $\left(\mathfrak{z}^{*}\right)$ in $D^{*}$ which is made up of a finite number of pairs:

$$
\left\{\left(\sigma_{i j}^{ \pm}, \mp e_{i j}\right)\right\}_{1 \leq i \leq m, 1 \leq j \leq n} \text { in } D^{*}
$$

where $\mp e_{i j}$ is the vanishing order of $\sigma_{i j}^{ \pm}$, respectively.

Consider a Cousin II distribution $\left((\mathfrak{z}) \cdot\left(\mathfrak{z}^{*}\right) \cdot\left(\mathfrak{z}^{*}\right)\right)$ in $D^{*}$. By applying Lemma 3.2 to the domain $D_{0} \Subset D^{*}$, there exists a continuous function $f$ in some neighborhood $V$ of $\left[\left((\mathfrak{z}) \cdot\left(\mathfrak{z}^{*}\right) \cdot\left(\mathfrak{z}^{*}\right)\right)\right] \mid D_{0}$ such that $f=0$ in $D_{0}$ is $\left((\mathfrak{z}) \cdot\left(\mathfrak{z}^{*}\right) \cdot\left(\mathfrak{z}^{*}\right)\right) \mid D_{0}$. Note that $I\left(\left((\mathfrak{z}) \cdot\left(\mathfrak{z}^{*}\right) \cdot\left(\mathfrak{z}^{*}\right)\right), \Gamma_{i j}\right)=-e_{i j}$ in $D^{*}$. Since $\left[\left((\mathfrak{z}) \cdot\left(\mathfrak{z}^{*}\right) \cdot\left(\mathfrak{z}^{*}\right)\right)\right]$ is analytic surfaces in the cylindrical domain $D^{*}$, we see (from [6, No. 1, p. 59]) that there exists a holomorphic polyhedron $G$ in $D^{*}$ such that $\left[\left((\mathfrak{z}) \cdot\left(\mathfrak{z}^{*}\right) \cdot\left(\mathfrak{z}^{*}\right)\right)\right] \mid D_{0} \Subset G \Subset V$. Hence, by Oka's principle ([3, p. 94]), there exists a holomorphic function $F$ in $G$ with the given zero $\left((\mathfrak{z}) \cdot\left(\mathfrak{z}^{*}\right) \cdot\left(\mathfrak{z}^{*}\right)\right) \mid G$.

We define a Cousin II distribution $(\tilde{\mathfrak{z}})=\left\{(F-t, G),\left(1, D_{0} \backslash G\right)\right\}$ in $D_{0}$, where $0<t \ll 1$. Then $[(\tilde{\mathfrak{z}})] \cap\left[\left((\mathfrak{z}) \cdot\left(\mathfrak{z}^{*}\right) \cdot\left(\mathfrak{z}^{*}\right)\right)\right]=\varnothing$ by $0<t$, and $I\left((\tilde{\mathfrak{z}}), \Gamma_{i j}\right)=-e_{i j}$ in $D_{0}$ by $t \ll 1$. Since $I\left(((\tilde{\mathfrak{z}}) \cdot(\mathfrak{z})), \Gamma_{i j}\right)=0$ in $D_{0}$, it follows from the sufficient condition of Proposition 2.4 that $((\tilde{\mathfrak{z}}) \cdot(\mathfrak{z}))$ has a solution of the Cousin II problem in $D_{0}$. Moreover, the result of the construction is $[(\tilde{\mathfrak{z}})] \cap[(\mathfrak{z})]=\emptyset$. Thus, we have proved case (i) in Theorem 1.

We shall construct a specific example for case (ii) in Theorem 1 by modifying Oka's example ([4, p. 10]).

Example. In $\mathbb{C}^{3}=\mathbb{C}_{x} \times \mathbb{C}_{y} \times \mathbb{C}_{z}$, we consider the cylindrical domain $D:=$ $D_{1} \times D_{2} \times D_{3}$

$$
D_{1}: r_{1}<|x|<1, \quad D_{2}: r_{2}<|y|<1, \quad D_{3}:|z|<1,
$$

where $r_{1}+r_{2}>1$. Let $\sigma$ be an analytic surface $x-y-1=0$ in $D$. Since $\sigma \cap\{\Im x=0\}=\emptyset, \sigma$ is divided into two disjoint parts $\sigma^{+}$and $\sigma^{-}$such 
that $\sigma^{+}=\sigma \cap\{(x, y, z) \in D \mid \mathfrak{I} x>0\}$ and $\sigma^{-}=\sigma \cap\{(x, y, z) \in D \mid \mathfrak{I} x<0\}$. Put $U_{1}:=D \backslash \sigma^{-}$and $U_{2}:=D \backslash \sigma^{+}$. If we set

$$
\begin{gathered}
f_{1}(x, y, z)=(x-y-1) \cdot z \quad \text { in } U_{1}, \\
f_{2}(x, y, z)=z \quad \text { in } U_{2},
\end{gathered}
$$

then $(\mathfrak{z})=\left\{\left(f_{1}, U_{1}\right),\left(f_{2}, U_{2}\right)\right\}$ defines a Cousin II distribution in $D$ (because $D=$ $U_{1} \cup U_{2}$ and $f_{1} / f_{2}=x-y-1 \neq 0$ in $\left.U_{1} \cap U_{2}\right)$. Thus, $[(\mathfrak{z})]=\sigma^{+} \cup\{z=0\}$ in $D$. Then there is no solution of the extra zero problem for (z) in $D$.

Proof. Assume that a solution $F$ of the extra zero problem does exist for (z) in $D$. That is, the holomorphic function $F$ in $D$ has an extra zero $\left(\mathfrak{z}^{*}\right)$ such that $\left[\left(\mathfrak{z}^{*}\right)\right] \cap$ $[(\mathfrak{z})]=\emptyset$. Take a closed cylindrical domain $D^{\prime}:=\left\{r_{1}^{\prime} \leq|x| \leq r_{1}^{\prime \prime}\right\} \times\left\{r_{2}^{\prime} \leq\right.$ $\left.|y| \leq r_{2}^{\prime \prime}\right\} \times\left\{|z| \leq r_{3}\right\}$ in $D$, where $r_{i}<r_{i}^{\prime}<r_{i}^{\prime \prime}<1(i=1,2)$ and $r_{3}<1$. Since $\left[\left(\mathfrak{z}^{*}\right)\right] \cap\{z=0\}=\emptyset$, we can find a small positive number $\varepsilon>0$ such that $\left(\left[\left(\mathfrak{z}^{*}\right)\right] \mid D^{\prime}\right) \cap\{|z| \leq \varepsilon\}=\emptyset$. Let $\gamma_{1}$ and $\gamma_{2}$ be circles (counterclockwise) $|x|=c_{1}$ and $|y|=c_{2}\left(r_{i}^{\prime}<c_{i}<r_{i}^{\prime \prime} ; i=1,2\right)$, respectively, and put $\Gamma_{12}:=\gamma_{1} \times \gamma_{2}$. Fix $z=\varepsilon$. The set $[(\mathfrak{z})(\varepsilon)] \cap \Gamma_{12}$ consists of one point in $\mathbb{C}_{x} \times \mathbb{C}_{y}$, and the real one-dimensional curve $\gamma_{2}+1$ in $\mathbb{C}_{x}$ traverses $\gamma_{1}$ at the point from the right to the left. Thus, $I\left((\mathfrak{z})(\varepsilon), \Gamma_{12}\right)=+1$. On the other hand, from the necessary condition in Proposition $2.4, I\left(\left((\mathfrak{z}) \cdot\left(\mathfrak{z}^{*}\right)\right)(\varepsilon), \Gamma_{12}\right)$ must be 0 . Thus, $I\left(\left(\mathfrak{z}^{*}\right)(\varepsilon), \Gamma_{12}\right)=-1$ and, hence, $\left(\left[\left(\mathfrak{z}^{*}\right)\right] \mid D^{\prime}\right) \cap\{z=\varepsilon\} \neq \emptyset$. This is a contradiction.

In the case $n \geq 4$, we can also make a counterexample of the extra zero problem in cylindrical domains in $\mathbb{C}^{n}$ in a similar way.

\section{Proof of Theorem 2}

Proof. Let $D$ and (z) satisfy the conditions in Theorem 2. Then $G=D \backslash[(\mathfrak{z})]$ is a domain of holomorphy (see [1, Satz 1]).

We shall prove that $G$ is not rationally convex in $\mathbb{C}^{n}$. Assume that $G$ is rationally convex. By the assumption and Proposition 2.4, for some homotopy classes $\left[\gamma_{k}\right]$ of simple closed curves in $D_{k}(k=i, j)$, there exist some smooth representatives $\gamma_{k}(k=i, j)$ such that $\Gamma_{i j}=\gamma_{i} \times \gamma_{j}$ is transversal to $(\mathfrak{z})(p)$ for some $p \in D^{(i j)}$ in general position with respect to $(\mathfrak{z})$, and $I\left((\mathfrak{z}),\left[\gamma_{i}\right],\left[\gamma_{j}\right]\right) \neq 0$. Let $Q^{\mu}=\left(z_{i}^{\mu}, z_{j}^{\mu}\right)(\mu=1, \ldots, l)$ be the intersection points of $\Gamma_{i j}$ and $[(\mathfrak{z})(p)]$, and let $e$ be the vanishing order of irreducible $[(\mathfrak{z})]$ in $D$. Then, from Definition 2.2, $I\left((\mathfrak{z}),\left[\gamma_{i}\right],\left[\gamma_{j}\right]\right)=I\left((\mathfrak{z})(p), \Gamma_{i j}\right)=\sum_{1 \leq \mu \leq l} s\left([(\mathfrak{z})(p)], \Gamma_{i j}^{Q^{\mu}}\right) \cdot e$. Thus, $m:=$ $\sum_{1 \leq \mu \leq l} s\left([(\mathfrak{z})(p)], \Gamma_{i j}^{Q^{\mu}}\right) \neq 0$. 
We divide the proof into two steps.

(1) We may assume that $z_{j}^{\mu} \neq z_{j}^{\nu}(\mu \neq v ; \mu, v=1, \ldots, l)$. Let $U_{i}^{\mu} \times U_{j}^{\mu}$ and $\varphi^{\mu}: U_{j}^{\mu} \rightarrow U_{i}^{\mu}$ satisfy the conditions in Definition 2.2. We may also assume that $U_{j}^{\mu} \cap U_{j}^{v}=\varnothing(\mu \neq v)$. Given $0<\eta \ll 1$, we consider an $\eta$-tubular neighborhood $A_{i} \times A_{j}$ of $\Gamma_{i j}$, where $A_{k}=\left\{z_{k} \in D_{k} \mid \operatorname{dist}\left(z_{k}, \gamma_{k}\right) \leq \eta\right\}(k=i, j)$. Choose $\eta>0$ so small that $\mathbf{s}:=[(\mathfrak{z})(p)] \cap\left(A_{i} \times A_{j}\right)$ is the analytic surfaces in $A_{i} \times A_{j}$ which consist of $l$ disjoint irreducible components $\mathbf{s}^{\mu}(\mu=1, \ldots, l)$ such that $\mathbf{s}^{\mu} \Subset \varphi^{\mu}\left(U_{j}^{\mu}\right) \times U_{j}^{\mu} \subset U_{i}^{\mu} \times U_{j}^{\mu}$ and the projection of $\mathbf{s}^{\mu}$ to $\mathbb{C}_{z_{j}}$ is diffeomorphic to a rectangle whose facing two sides are contained in $\left(\partial A_{j}\right) \cap U_{j}^{\mu}$ and other two sides are mapped by $\varphi^{\mu}$ onto two curves in $\left(\partial A_{i}\right) \cap U_{i}^{\mu}$. We simply put $A_{i} / 2=\left\{z_{i} \in D_{i} \mid \operatorname{dist}\left(z_{i}, \gamma_{i}\right) \leq \eta / 2\right\}$ and $\mathbf{s} / 2:=[(\mathfrak{z})(p)] \cap\left(A_{i} / 2 \times A_{j}\right)$. Let $\gamma_{j}^{\mu}:=$ $\left\{z_{j} \in \gamma_{j} \cap U_{j}^{\mu} \mid\left(\varphi^{\mu}\left(z_{j}\right), z_{j}\right) \in \mathbf{s}\right\}, \gamma_{j}^{\mu} / 2:=\left\{z_{j} \in \gamma_{j} \cap U_{j}^{\mu} \mid\left(\varphi^{\mu}\left(z_{j}\right), z_{j}\right) \in \mathbf{s} / 2\right\}$ and $C_{j}^{\mu}:=\left\{\left(\varphi^{\mu}\left(z_{j}\right), z_{j}\right) \in \mathbf{s} \mid z_{j} \in \gamma_{j}^{\mu}\right\}$ for $\mu=1, \ldots, l$. By the assumption, (z) necessarily satisfies Weierstrass' condition for $z_{i}$ at any point of $[(\mathfrak{z})]$. We thus let $\pi:[(\mathfrak{z})] \rightarrow \mathbb{C}^{(i)}=\mathbb{C}_{z_{1}} \times \cdots \times \hat{\mathbb{C}}_{z_{i}} \times \cdots \times \mathbb{C}_{z_{n}}$ be the analytic projection, whose image $\mathcal{R}:=\pi([(\mathfrak{z})])$ is a Riemann domain over $D^{(i)}$ with branch surfaces. We connect $l$ points $\left(Q^{\mu}, p\right)(\mu=1, \ldots, l)$ with a simple smooth curve $\mathcal{L}$ in $[(\mathfrak{z})]$, and put $L:=\pi(\mathcal{L})$. If necessary, move $\gamma_{i}, \gamma_{j}$ and $\mathcal{L}$ slightly, we may assume that, for each $\mu=1, \ldots, l$ :

(i) $\mathcal{L} \cap\left(C_{j}^{\mu} \times p\right)=\left\{\left(Q^{\mu}, p\right)\right\}$;

(ii) any $C_{j}^{\mu} \times p$ and $\mathcal{L}$ do not pass the singular sets of [(z) ];

(iii) any $\gamma_{j}^{\mu} \times p$ and $L$ do not pass the branch surfaces of $\mathcal{R}$.

Put $K:=L \cup\left(\bigcup_{\mu=1}^{l} \gamma_{j}^{\mu} \times p\right)(\subset \mathcal{R})$. From (i)-(iii), we uniquely have a continuous function $\psi: K \rightarrow \mathbb{C}_{z_{i}}$ such that $\left(\psi\left(z^{(i)}\right), z^{(i)}\right) \in[(\mathfrak{z})]$ for $z^{(i)} \in K$ and such that $\psi\left(\zeta_{1}\right) \neq \psi\left(\zeta_{2}\right)$ if $\zeta_{1} \neq \zeta_{2}$ as points in $\mathcal{R}$.

Fix two cylindrical domains $D^{\prime} \Subset D^{\prime \prime} \Subset D$ with $\left(A_{i} \times A_{j} \times p\right) \cup \mathcal{L} \Subset D^{\prime}$. Put $D^{\prime}=\prod_{k=1}^{n} D_{k}^{\prime}$ and $D^{\prime \prime}=\prod_{k=1}^{n} D_{k}^{\prime \prime}$. By the assumption, we obtain a meromorphic function $g$ in $D^{\prime \prime}$ whose pole is only on [(z) $]$. Consider a continuous function $h=1 /|g|$ in $D^{\prime \prime}$. Then $h$ is class $C^{\omega}$ on $D^{\prime \prime} \backslash[(\mathfrak{z})]$ such that $h \geq 0$ on $D^{\prime \prime}$ and $h=0$ if and only if $z \in[(\mathfrak{z})] \cap D^{\prime \prime}$. Given $\varepsilon>0$, we define the following tubular neighborhood of $[(\mathfrak{z})] \cap D^{\prime \prime}$ in $D^{\prime \prime}$ :

$$
V_{\varepsilon}:=\left\{z \in D^{\prime \prime} \mid h(z)<\varepsilon\right\} \quad\left(\supset[(\mathfrak{z})] \cap D^{\prime \prime}\right) .
$$

For each $z^{(i)} \in K$, we define $V_{\varepsilon}\left(z^{(i)}\right):=\left\{z_{i} \in D_{i}^{\prime \prime} \mid\left(z_{i}, z^{(i)}\right) \in V_{\varepsilon}\right\}$. We denote by $v_{\varepsilon}\left(z^{(i)}\right)$ the connected component of $V_{\varepsilon}\left(z^{(i)}\right)$ containing $\psi\left(z^{(i)}\right)$, and put $r_{\varepsilon}\left(z^{(i)}\right):=$ $\max \left\{\left|z_{i}-\psi\left(z^{(i)}\right)\right| \mid z_{i} \in \partial v_{\varepsilon}\left(z^{(i)}\right)\right\}>0$ so that $\lim _{\varepsilon \rightarrow 0} r_{\varepsilon}\left(z^{(i)}\right)=0$. We note that, 
for $z^{(i)}=z_{j} \times p \in K$ where $z_{j} \in \gamma_{j}^{\mu} / 2$, the set $\left\{z_{i} \in D_{i}^{\prime \prime} \mid\left(z_{i}, z^{(i)}\right) \in[(\mathfrak{z})]\right\}$ consists of finite points: $\psi\left(z^{(i)}\right)\left(=\varphi^{\mu}\left(z_{j}\right)\right)$ and $z_{i v}(v=1, \ldots, N)$ such that $z_{i v} \notin \gamma_{i}$.

Then we find a sufficiently small $\varepsilon>0$ which satisfies the following conditions: (e1) $r_{\varepsilon}\left(z^{(i)}\right)<\eta / 4$ for each $z^{(i)} \in K$;

(e2) $\min \left\{h\left(z_{i}, z_{j}, p\right) \mid\left(z_{i}, z_{j}\right) \in \gamma_{i} \times\left(\gamma_{j} \backslash \bigcup_{\mu=1}^{l} \gamma_{j}^{\mu} / 2\right)\right\}>\varepsilon$;

(e3) each $v_{\varepsilon}\left(z^{(i)}\right)\left(z^{(i)} \in K\right)$ is bounded by a simple smooth closed curve $\partial v_{\varepsilon}\left(z^{(i)}\right)$ in $D_{i}^{\prime}$ and $\partial v_{\varepsilon}\left(z^{(i)}\right)$ varies continuously in $D_{i}^{\prime}$ with parameter $z^{(i)} \in K$;

(e4) $\left(V_{\varepsilon}\left(z^{(i)}\right) \backslash v_{\varepsilon}\left(z^{(i)}\right)\right) \cap \gamma_{i}=\emptyset$ for $z^{(i)}=z_{j} \times p$ where $z_{j} \in \gamma_{j}^{\mu} / 2(\mu=1, \ldots, l)$;

(e5) we fix one point $\left(\psi\left(z_{0}^{(i)}\right), z_{0}^{(i)}\right)$, where $z_{0}^{(i)} \in K$, and then

$$
\min \left\{\left|g\left(z_{i}, z_{0}^{(i)}\right)\right| \mid z_{i} \in \partial v_{\varepsilon}\left(z_{0}^{(i)}\right)\right\}>\max \left\{\left|g\left(z_{i}, z_{0}^{(i)}\right)\right| \mid z_{i} \in \partial D_{i}^{\prime}\right\} .
$$

Since $D^{\prime \prime} \backslash[(\mathfrak{z})]$ is rationally convex in $\mathbb{C}^{n}$, it follows from $D^{\prime} \backslash V_{\varepsilon} \Subset D^{\prime \prime} \backslash[(\mathfrak{z})]$ that, given $0<\delta \ll 1$, there exists a rational function $\mathfrak{P} / \mathfrak{Q}$ in $\mathbb{C}^{n}$ (where $\mathfrak{P}, \mathfrak{Q}$ are relatively prime) such that $|g-\mathfrak{P} / \mathfrak{Q}|<\delta$ on $D^{\prime} \backslash V_{\varepsilon}$. By the usual argument about the points of indeterminancy,

$$
\mathfrak{Q}(z) \text { does not vanish in } D^{\prime} \backslash V_{\varepsilon} \text {. }
$$

If we take $\delta: 0<\delta \ll 1$ so small, then (e5) implies $\min \left\{\left|\mathfrak{P} / \mathfrak{Q}\left(z_{i}, z_{0}^{(i)}\right)\right| \mid z_{i} \in\right.$ $\left.\partial v_{\mathcal{\varepsilon}}\left(z_{0}^{(i)}\right)\right\}>\max \left\{\left|\mathfrak{P} / \mathfrak{Q}\left(z_{i}, z_{0}^{(i)}\right)\right| \mid z_{i} \in \partial D_{i}^{\prime}\right\}$. It follows from the maximum modulus principle that $\mathfrak{Q}\left(z_{i}, z_{0}^{(i)}\right)$ (as a function of $z_{i} \in \mathbb{C}_{z_{i}}$ ) has at least one zero in $v_{\varepsilon}\left(z_{0}^{(i)}\right)$, say $\lambda$, the number of zeros with multiplicity of $\mathfrak{Q}\left(z_{i}, z_{0}^{(i)}\right)$. For any fixed $z^{(i)} \in K$, we have $\mathfrak{Q}\left(z_{i}, z^{(i)}\right) \neq 0$ on $\partial v_{\varepsilon}\left(z^{(i)}\right)$ by (4.1). Under this condition and (e3), Weierstrass preparation theorem implies that the number of zeros of $\mathfrak{Q}\left(z_{i}, z^{(i)}\right)$ in $v_{\varepsilon}\left(z^{(i)}\right)$ is independent as long as $z^{(i)}$ runs along $K$, i.e. is always equal to $\lambda$.

(2) We simply put $\mathfrak{Q}_{0}\left(z_{i}, z_{j}\right):=\mathfrak{Q}\left(z_{i}, z_{j}, p\right)$ and $\left(\mathcal{Z}_{0}\right):=\left\{\left(z_{i}, z_{j}\right) \in A_{i} \times A_{j} \mid\right.$ $\left.\mathfrak{Q}_{0}\left(z_{i}, z_{j}\right)=0\right\}$. Then we prove that $I\left(\left(\mathcal{Z}_{0}\right), \gamma_{i} \times \gamma_{j}^{\mu} / 2\right)=s\left([(\mathfrak{z})(p)], \Gamma_{i j}^{Q^{\mu}}\right) \cdot \lambda$ for $\mu=1, \ldots, l$. In fact, for every $z_{j} \in \gamma_{j}^{\mu}(\mu=1, \ldots, l)$, we put $v_{\varepsilon}\left(z_{j}\right):=$ $v_{\varepsilon}\left(z_{j}, p\right)$. Then (4.1) and (e2) imply that $\mathfrak{Q}_{0}\left(z_{i}, z_{j}\right) \neq 0$ on $\bigcup_{z_{j} \in \gamma_{j}^{\mu} / 2}\left(\gamma_{i} \backslash v_{\varepsilon}\left(z_{j}\right), z_{j}\right)$. We see from (e1) that $\gamma_{i} \cap v_{\varepsilon}\left(z_{j}\right)=\emptyset$ for every $z_{j} \in \gamma_{j}^{\mu} \backslash \gamma_{j}^{\mu} / 2$. Hence, $\left[\left(\mathcal{Z}_{0}\right)\right] \cap\left(\gamma_{i} \times\left(\gamma_{j}^{\mu} \backslash\left(\gamma_{j}^{\mu} / 2\right)\right)\right)=\emptyset$.

Now consider the case when $s\left([(\mathfrak{z})(p)], \Gamma_{i j}^{Q^{\mu}}\right)=+1$ : We have $\psi\left(z_{j}, p\right)=$ $\varphi^{\mu}\left(z_{j}\right)$ for $z_{j} \in \gamma_{j}^{\mu}$. Let the subarc $\gamma_{j}^{\mu} / 2$ of $\gamma_{j}$ (with same orientation as $\gamma_{j}$ ) have initial point $a^{\mu}$ and terminal point $b^{\mu}$. Note that $v_{\varepsilon}\left(a^{\mu}\right)\left(\subset A_{i}\right)$ contains $\lambda$ zeros of $\mathfrak{Q}_{0}\left(z_{i}, a^{\mu}\right)$, all of these $\lambda$ zeros lie in the right-hand side of $\gamma_{i}$ from (e1). On the other hand, $v_{\varepsilon}\left(b^{\mu}\right)\left(\subset A_{i}\right)$ contains $\lambda$ zeros of $\mathfrak{Q}_{0}\left(z_{i}, b^{\mu}\right)$, all of which lie in the left-hand 
side of $\gamma_{i}$. If $z_{j}$ moves along the oriented arc $\gamma_{j}^{\mu} / 2$, then the center $\varphi^{\mu}\left(z_{j}\right)$ of $v_{\varepsilon}\left(z_{j}\right)$ crosses $\gamma_{i}$ from the right to the left passing through $z_{i}^{\mu}\left(=\varphi^{\mu}\left(z_{j}^{\mu}\right)\right)$. This and (e4) imply that $I\left(\left(\mathcal{Z}_{0}\right), \gamma_{i} \times \gamma_{j}^{\mu} / 2\right)=+\lambda$. In the case when $s\left([(\mathfrak{z})(p)], \Gamma_{i j}^{Q^{\mu}}\right)=-1$, we similarly have $I\left(\left(\mathcal{Z}_{0}\right), \gamma_{i} \times \gamma_{j}^{\mu} / 2\right)=-\lambda$.

Since $\left[\left(\mathcal{Z}_{0}\right)\right] \cap\left(\gamma_{i} \times\left(\gamma_{j} \backslash \bigcup_{\mu=1}^{l} \gamma_{j}^{\mu} / 2\right)\right)=\emptyset$, we finally get

$$
I\left(\left(\mathcal{Z}_{0}\right), \gamma_{i} \times \gamma_{j}\right)=\sum_{\mu=1}^{l} I\left(\left(\mathcal{Z}_{0}\right), \gamma_{i} \times \frac{\gamma_{j}^{\mu}}{2}\right)=\lambda \sum_{\mu=1}^{l} s\left([(\mathfrak{z})(p)], \Gamma_{i j}^{Q^{\mu}}\right)=\lambda m .
$$

This contradicts Proposition 2.4, so that $G$ is not rationally convex.

Acknowledgements. The author would like to offer hearty thanks to Professors Toshio Nishino and Hiroshi Yamaguchi for invaluable discussions and advice, and also thank Professors Tetsuo Ueda and Kang-Tae Kim for useful comments. The author would like to express her gratitude to the referee who read the manuscript with great care and gave very useful comments and constructive suggestions for her future work. This research was partially supported by Yoshida Scholarship Foundation.

\section{REFERENCES}

[1] F. Docquier and H. Grauert. Levisches Problem und Rungescher Satz für Teilgebiete Steinscher Mannigfaltigkeiten. Math. Ann. 140 (1960), 94-123.

[2] H. Grauert. Charakterisierung der holomorph vollständigen komplexen Räume. Math. Ann. 129 (1955), 233-259.

[3] T. Nishino. Function Theory in Several Complex Variables (Translations of Mathematical Monographs, 193). American Mathematical Society, Providence, RI, 2001.

[4] K. Oka. Sur les fonctions analytiques de plusieurs variables, III Deuxième problème de Cousin. J. Sci. Hiroshima Univ. A9 (1939), 7-19.

[5] K. Oka. Sur les fonctions analytiques de plusieurs variables, IV Domaines d'holomorphie et Domaines rationnellement convexes. Japan. J. Math. 17 (1941), 517-521.

[6] K. Oka. Posthumous Papers of Kiyoshi Oka, Nos 1-7. Eds. T. Nishino and A. Takeuchi. Imperial University, Kyoto, 1980-1983.

[7] K. Stein. Topologische Bedingungen für die Existenz analytischer Funktionen komplexer Veränderlichen zu vorgegebenen Nullstellenflächen. Math. Ann. 117 (1941), 727-757.

[8] K. Stein. Analytische Funktionen mehrerer komplexer Veränderlichen zu vorgegebenen Periodizitätsmoduln und das zweite Cousinsche Problem. Math. Ann. 123 (1951), 201-222.

[9] M. Tsuji. Potential Theory in Modern Function Theory. Chelsea, New York, 1975. 
Sachiko Hamano

Department of Mathematics

Nara Women's University

Kitauoya-Nishimachi

Nara 630-8506

Japan

(E-mail: yas.hamano@cc.nara-wu.ac.jp) 